\title{
Monatliches Fortbildungscurriculum der Deutschen Gesellschaft für Neuroradiologie (DGNR)
}

\section{Sie haben ein für 서영}

\author{
Dann schauen Sie rein - bei \\ Fit für Neuroradiologie \\ Ihrganzjähriges Online-Curriculum
}

Liebe Kolleginnen und Kollegen,

liebe (Neu-)Mitglieder unserer Fachgesellschaft,

wir freuen uns, Ihnen zum Jahresbeginn 2022 ein neues Fortbildungskonzept vorstellen zu dürfen.

Das Fortbildungscurriculum „Fit für Neuroradiologie“ ist eine ganzjährige zoombasierte Webinar-Reihe, die in monatlichen Veranstaltungen relevante Inhalte der gesamten Neuroradiologie abhandeln wird.

Wir möchten mit dieser Reihe gleichermaßen Weiterbildungsassistent*innen der Radiologie, angehende Schwerpunktneuroradiolog*innen, Facharztkandidaten aus den Nachbardisziplinen (unter anderem Neurologie, Neurochirurgie, Psychiatrie), sowie alle anderen an unserem Fach Interessierte ansprechen. Aber auch versierte
Neuroradiolog*innen profitieren von dem Programm, der Gestaltung und den exzellenten Referent*innen.

Online auf www.neuroradiologie.de finden Sie alle wichtigen Informationen zum Programmablauf, den Referent*innen und Terminen - bitte informieren Sie auch Ihre Kolleg*innen über dieses Angebot.

Wir freuen uns über zahlreiche Teilnahme!

Mit kollegialen Grüßen

Prof. Dr. Martin Wiesmann und Prof. Dr. Jennifer Linn

Programmkoordinator*innen

Prof. Dr. Claus Zimmer

Präsident der DGNR 2020-2022

Dr. Daniel Kaiser

Sprecher der Jungen Neuroradiologie

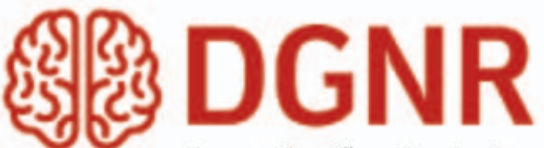

Deutsche Gesellschaft für Neuroradiologie e.V.

\section{Fit für Neuroradiologie - kostenfrei für DGNR-Mitglieder!}

Das Gesamtpaket mit allen Veranstaltungen à 90 Minuten ist für DGNR-Mitglieder kostenfrei.

Anmeldung \& weitere Informationen: www.neuroradiologie.de > Fachinfos $>$ Fit für Neuroradiologie

\section{Noch kein Mitglied?}

Einmalige Aufnahmegebühr von $100 €$ für Ärzt*innen in Weiterbildung. Beantragen Sie hier Ihre Mitgliedschaft: www.neuroradiologie.de > Über uns > Mitgliedschaft

Im Februar erwartet Sie das Thema: Sinus- und Hirnvenenthrombosen Referentin: Prof. Dr. Linn, Universitätsklinikum Dresden

Zeit: 16.02.2022, 17:30-19:00

Ort: Zoom (den Link erhalten Sie nach der Anmeldung)

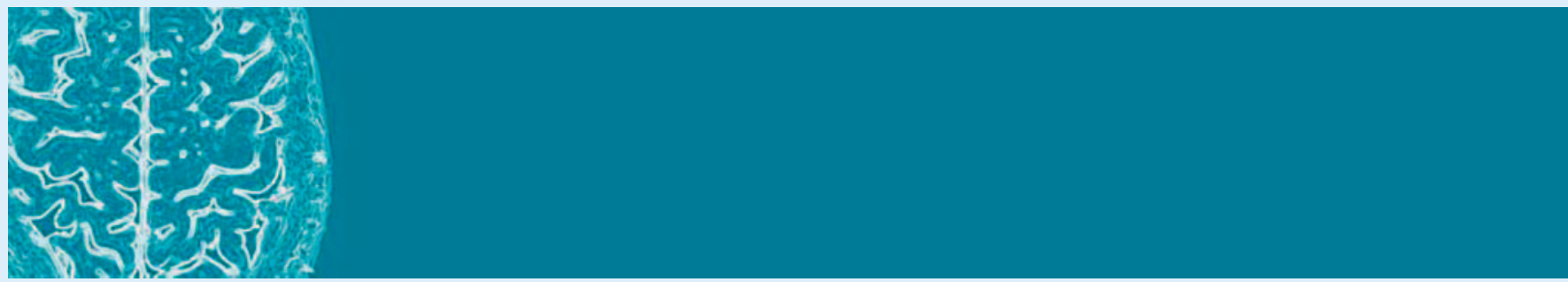

